\title{
A cocktail of antibodies to multiple tumor-specific neoantigens increases binding and inhibits tumor growth
}

\section{Girja S. Shukla}

University of Vermont Larner College of Medicine

\section{Yu-Jing Sun}

University of Vermont Larner College of Medicine

Stephanie C. Pero

University of Vermont Larner College of Medicine

David N. Krag ( $\sim$ David.Krag@uvm.edu )

University of Vermont College of Medicine https://orcid.org/0000-0001-5355-5999

Research article

Keywords: Tumor-specific, neoantigens, antibodies, tumor inhibition, mouse model, melanoma

Posted Date: June 17th, 2019

DOl: https://doi.org/10.21203/rs.2.10383/v1

License: (c) (i) This work is licensed under a Creative Commons Attribution 4.0 International License.

Read Full License 


\section{Abstract}

Background: Antibodies that target a single tumor antigen fail to cure stage IV cancer patients due to tumor heterogeneity resulting in variable expression of antigen. Tumor cells with insufficient binding of antibody will not undergo antibody induced cytotoxicity. We describe targeting multiple tumor-specific antigens that resulted in homogeneous dense binding to mouse melanoma cells and significant tumor growth inhibition. Methods: Surface-related tumor-specific mutations on B16-F10 cells were identified. Peptides containing the single amino acid mutation were synthesized for 9 different neoantigens. Rabbits were vaccinated with each of these peptides and high affinity polyclonal antibodies to each peptide were obtained. The 9 antibodies were combined as a cocktail and mice with implanted B16-F10 cells were treated with and without PD1 inhibitor. Results: Even a single dose of the antibody cocktail inhibited tumor growth and prolonged survival. PD1 inhibitor alone had little effect on tumor growth. The antibody cocktail plus PD1 inhibition increased tumor response and 4 doses of the cocktail completely prevented tumor growth in $50 \%$ of the mice. Complete responses were durable. The complete responders were highly resistant to tumor re-challenge at 6 months. No adverse events were identified in the antibody treated mice. Conclusions: Multiple tumor-specific cell surface-related neoantigens were abundant in B16F10 cells. Antibodies to 9 of these neoantigens had variable binding but when combined had dense homogeneous binding. Even one dose of this cocktail of 9 antibodies improved survival and when multiple does were combined with PD1 inhibition $50 \%$ of the mice were rendered permanently tumor free.

\section{Background}

Current antibody therapy for cancer is largely based on a single antibody binding to a single overexpressed antigen. The unresolved problem with this approach is that within the heterogeneous cancer cell population there are cancer cells with low antigen expression. These cells will not bind sufficient antibody and will continue to grow. This explains why Herceptin, considered to be a prototype blockbuster antibody, only delays time to progression by 3 months. None of the patients are cured [1].

Antibodies are highly capable of initiating cytotoxicity of target cells. Decades ago it was shown that serum transfer from animals resistant to a tumor would induce tumor resistance in a recipient animal [27]. Superb electron and fluorescent microscopic images graphically demonstrated multiple types of effector cells engaged in antibody directed cell destruction $[8,9]$. Target heterogeneity was understood as early as 1962, and it was shown that resistance to antibodies could be overcome by mixtures of antisera demonstrating that "cytotoxic sensitivity is related to the surface concentration of reactive sites" [10]. Even a cocktail of 3 or 4 antibodies was shown to increase bioactivity against tumor cell lines [11]. After observing that combinations of monoclonal antibodies increased effectiveness the authors suggested that cocktails of antibodies may be useful in the clinic [12].

In this study, we have attempted to overcome tumor heterogeneity in the B16-F10 mouse model. We focused on surface related tumor-specific neoantigens. Such tumor-specific mutations are abundant and largely overlooked because they are highly variable between individuals, are not driver mutations, and 
they are not necessarily overexpressed. We generated rabbit antibodies to multiple tumor-specific neoantigens and combined them together as a cocktail. The cocktail substantially increased cell surface binding across the cell population. We demonstrate that this strategy overcomes tumor heterogeneity and results in substantial inhibition of tumor growth.

\section{Methods}

Cells and reagents: B16-F10 melanoma tumor cells (ATCC CRL-6475) were procured from American Type Culture Collection (ATCC, Manassas, VA). Dulbecco's Modified Eagle's Medium (DMEM) and Trypsin-EDTA solution were purchased from ATCC. All other reagents used in this study were of molecular or high purity grade. Rat anti-mouse PD1 (CD279) antibody (clone RMP1-14) and rat IgG2a isotype control of anti-PD1 antibody (clone 2A3) were purchased from Bio X cell (West Lebanon, NH). Alexa Fluor 568 conjugated goat-anti-rabbit antibody and cross-absorbed goat anti-mouse IgG $(\mathrm{H}+\mathrm{L})$-horseradish peroxidase (HRP) conjugate were procured from Life Technologies (Carlsbad, CA).

Neoantigens: Using sequence data of B16-F10 mouse melanoma cells [13], we selected multiple cellsurface-related mutated proteins (neoantigens) that had a single amino acid change. The peptide length was kept to 11 amino acids, keeping mutated amino acid in or near the center (Table 1). The peptides were analyzed for their B-cell response predictions [14] and immunogenicity by determining the homology of the selected mouse antigen epitope with the same in host species. Antibodies to the selected peptides were commercially supplied by GenScript (Piscataway, NJ).

Anti-neoantigen antibodies: The purified antibodies supplied by GenScript were titered against the mutated peptides for affinity estimation (EC50) using ELISA [15]. Following peptide immobilization in 96well plates, the wells were blocked with $1 \%$ BSA in PBS. The plates were washed 3 times with PBS and incubated with serially (2-fold) diluted purified antibodies. Wells were washed again 3 times and incubated with HRP-conjugated anti-rabbit IgG antibodies. The binding was monitored $450 \mathrm{~nm}$ with 3,3',5,5'-tetramethylbenzidine (TMB) reagent as an HRP substrate.

Immunofluorescence microscopy of anti-neoantigen antibodies binding to B16-F10 cells: B16-F10 whole cell slides and B16-F10 mouse tumor sections were fixed in 3\% paraformaldehyde and washed with PBS (3 times, 3 min each) for immunofluorescence microscopy by the method described previously [16]. Briefly, the B16-F10 tumor cells and sections were blocked with Image-it Fx signal enhancer (Invitrogen, Carlsbad, CA) and were incubated with individual polyclonal anti-mutated peptide antibodies and their cocktail for $1 \mathrm{~h}$ at room temperature. Slides were rinsed 3 times in PBS and incubated for 30 min with Alexa Fluor 568-conjugated goat anti-rabbit antibody (Invitrogen). The slides were rinsed again in PBS, 3 
times for 3 min each, and stained with 1000x diluted DAPI (Invitrogen) for $10 \mathrm{~min}$. The slides were coverslipped and examined (Ex 579 nm/Em 603 nm and Ex 359 nm/Em 461 nm) using Nikon TE2000-U inverted fluorescence microscope (Nikon Corp., Kangawa, Japan).

Animal tumor model: The spontaneous C57BL/6-derived B16-F10 melanoma tumor cells were used as a model system for this study. It is a well-established and widely used syngeneic tumor model representing an aggressive tumor that is difficult to treat. Six to seven weeks old (date of birth \pm 3 days) female mice of C57BL/6J strain were purchased from The Jackson Laboratory (Bar Harbor, ME). The mice were allowed to acclimate in the animal facility for one week before starting the experiment. The animal facility was maintained at the standard conditions with 12-h light/dark cycles. Mice received food pellets and water ad libitum. B16-F10 melanoma tumor cells were cultured to $70 \%$ confluency in DMEM containing $10 \%(\mathrm{v} / \mathrm{v})$ fetal bovine serum following supplier's suggestions. The cells were confirmed negative for mycoplasma infection prior to implantation. The cells were washed in PBS and subcutaneously injected at the shaved right flank of the mice. All animal procedures in the present study were approved (Protocol \# 18-002) by the University of Vermont Institutional Animal Care and Use Committee (UVM IACUC).

\section{Animal treatments:}

Experiment 1: The goal was to determine the optimal number of B16-F10 cells inoculation for producing a tumor that grows to $\sim 2000 \mathrm{~mm}^{3}$ size in 15-20 days. For this, 4 groups of mice subcutaneously received $1 \times 10^{4}, 3 \times 10^{4}, 1 \times 10^{5}$ and $3 \times 10^{5}$ B16-F10 melanoma tumor cells suspended in PBS and the tumor size was measured.

Experiment 2: This experiment was conducted to determine the effect of anti-neoantigen rabbit polyclonal antibodies treatment on the B16-F10 melanoma tumor growth and survival. The animals received subcutaneous implantation of $3 \times 10^{5}$ B16-F10 melanoma tumor cells. The mice were divided into 5 groups and treated as follows:

Gr 1. PBS Control: Mice received B16-F10 cells implantation in PBS.

Gr 2. Ig Control-1 Dose: Mice received B16-F10 cells implantation in $0.2 \mathrm{mg}$ rabbit Ig (no further treatment).

Gr 3. Ab Cocktail-1 Dose: Mice received B16-F10 cells implantation in 0.2 mg cocktail of 9 anti-mutated peptides antibodies (no further treatment).

Gr 4. Ig Control-4 Doses: Mice received B16-F10 cells implantation in PBS + subcutaneous $0.2 \mathrm{mg}$ rabbit Ig at the base of tumor on 3, 6, 9 and 12 days post implantation (DPI). 
Gr 5. Ab Cocktail-1 Doses: Mice received B16-F10 cells implantation in PBS + subcutaneous $0.2 \mathrm{mg}$ cocktail of 9 anti-mutated peptides antibodies at the base of tumor on 3, 6, 9 and 12 DPI.

Experiment 3: This experiment was conducted to determine the effect of combined treatment of antineoantigen rabbit polyclonal antibodies and anti-PD1 antibody (PD1i) on the B16-F10 melanoma tumor growth and survival. A group of mice also received rat IgG2a isotype control of PD1i. The animals received subcutaneous implantation of $3 \times 10^{5}$ B16-F10 melanoma tumor cells. The mice were divided into 7 groups and treated as following:

Gr 1. PBS Control: Mice received B16-F10 cells implantation in PBS.

Gr 2. PBS+PD1i only: Mice received B16-F10 cells implantation in PBS + intraperitoneal $0.2 \mathrm{mg}$ PD1i on 3, 6,9 and $12 \mathrm{DPI}$.

Gr 3. Ig Control-1 Dose+PD1i: Mice received B16-F10 cells implantation in $0.2 \mathrm{mg}$ rabbit lg + intraperitoneal $0.2 \mathrm{mg}$ PD1i on 3, 6, 9 and $12 \mathrm{DPI}$.

Gr 4. Ab Cocktail-1 Dose+PD1i: Mice received B16-F10 cells implantation in 0.2 mg cocktail of 9 antimutated peptides antibodies + intraperitoneal 0.2 mg PD1i on 3, 6, 9 and 12 DPI.

Gr 5. Ig Control-4 Doses+PD1i: Mice received B16-F10 cells implantation in PBS + subcutaneous $0.2 \mathrm{mg}$ rabbit lg at the base of tumor and intraperitoneal $0.2 \mathrm{mg}$ PD1i, both treatments on 3, 6, 9 and $12 \mathrm{DPI}$.

Gr 6. Ab Cocktail-4 Doses+PD1i: Mice received B16-F10 cells implantation in PBS + subcutaneous $0.2 \mathrm{mg}$ cocktail of 9 anti-mutated peptides antibodies at the base of tumor and intraperitoneal $0.2 \mathrm{mg}$ PD1i, both treatments on $3,6,9$ and $12 \mathrm{DPI}$.

Gr 7. PBS+PD1 Isotype Control: Mice received B16-F10 cells implantation in PBS + intraperitoneal $0.2 \mathrm{mg}$ isotype control of PD1i antibody on 3, 6, 9 and 12 DPI.

Experiment 4: The surviving mice from Experiment 3 treated with 4 doses of antibody cocktail and PD1i were shaved and subcutaneously re-inoculated with $3 \times 10^{5}$ B16-F10 melanoma tumor cells second time after 6.5 months of first tumor challenge. A group of normal mice were also implanted with $3 \times 10^{5} \mathrm{~B} 16$ F10 melanoma tumor cells in the identical manner to serve as controls. Both the groups of mice were left without any treatment and their tumors were measured at different time intervals.

The animals of all the experiments were weighed 2 times/week and the tumor size were followed by measuring tumor volume $\left(\mathrm{V}=\mathrm{w}^{2} \mathrm{xl}_{2}\right)$ every day starting from 7 days post-implantation using an electronic caliper. The survival time was calculated based on the death of an animal or euthanized following reaching to $\geq 2000 \mathrm{~mm}^{3}$ tumor volume. Following our IACUC approved protocol, the tumorbearing animals were euthanized when tumor reached $\geq 2000 \mathrm{~mm}^{3}$ or when animals exhibit signs of dehydration, difficulty walking, cachexia or other signs of physical distress, whichever comes first. 
ELISA for binding of tumor-bearing mouse plasma antibodies to B16-F10 tumor lysate: The lysate tumor preparation and lysate ELISA were done by the methods described previously [17]. Briefly, the lysate of B16-F10 tumors grown in untreated mice were prepared by the cycles of freezing/thawing and ultrasonication. Following $50 \mu \mathrm{L}$ cell lysate $(1 \mathrm{mg} / \mathrm{mL}$ protein) immobilization and well-blockage with $1 \%$ casein-TBS (Thermo Fisher), diluted (100, 200 and 400x) plasma of normal and tumor bearing mice were added and incubated for $2 \mathrm{hr}$ at room temperature. The wells were washed and incubated with crossabsorbed goat anti-mouse IgG ( $\mathrm{H}+\mathrm{L}$ )-horseradish peroxidase (HRP) conjugate (Life Technologies, Carlsbad, CA). The lysate-bound plasma antibodies were detected with TMB soluble substrate (Calbiochem, Billerica, MA).

Statistical analyses: Tumor growth differences in the animals of different groups were analyzed by Oneway and Two-way ANOVA followed by Tukey's multiple comparison test. Kaplan Meier plot were used for survival data presentation and the survival curve comparisons for determining the significance of difference in groups were analyzed using Log-rank (Mantel-Cox) test and median survival values. Prism software (GraphPad, San Diego, CA) was used for some of the analyses.

\section{Results}

Polyclonal rabbit antibodies demonstrated high-affinity antibodies against each mutated peptide. Fig. 1 displays the results of characterization of these antibodies. Fig. 1A shows the titration results of serially diluted purified antibody samples against each mutated peptide. The lowest dilution of the antibodies plotted in Fig. 1A was 1000-fold dilution. The results demonstrated high titer levels $(\geq 1: 500,000)$ of these antibody samples. The EC50 values presented in Fig. 1B show picomolar level affinity of these rabbit antibodies.

Fluorescence microscopy of individual antibodies binding to B16-F10 tumor cells (Fig. 1C) and mouse tumor sections (results not shown) demonstrated positive binding by all the antibodies. The results show variation in the binding of individual antibodies to B16-F10 cells. Since all antibodies had high affinity, variation in cell binding was most likely associated with variation in target density. Pooling equal amounts of all 9 antibodies as a cocktail (far left panel of Fig. 1C) overcame the variation observed with individual antibodies and resulted in strong and homogeneous binding.

Following a one-week acclimation period in our animal facility and prior to any treatment, the weights of each animal were obtained for baseline measure.

Animal Experiment 1: The effects of inoculating different numbers of B16-F10 cells $(10,000-300,000)$ on tumor volume are presented in Supplementary Fig. S1. Tumor volumes increased more rapidly with higher numbers of tumor cells inoculated. Subcutaneous implantation of 300,000 B16-F10 cells produced 
very fast-growing tumors. By day $21 \mathrm{DPI}$, animals died or were euthanized because tumor size reached $2000 \mathrm{~mm}^{3}$. No unexpected weight changes were observed during this period. All treatment experiments used 300,000 tumor cells for implantation based on consistent rapid growth and lack of apparent side effects during tumor growth period. Also, during this short period of tumor growth, plasma antibodies to B16-F10 tumor lysates above background levels were not detectable (data not shown).

Animal Experiment 2: Tumor growth and mouse survival data following antibody cocktail treatment are presented in Fig. 2. The results demonstrate tumor growth inhibition by the treatment with the 9-antibody cocktail (Fig. 2A). Two-way ANOVA showed significant tumor growth differences by the treatment $(P<0.001)$ and time $(P<0.001)$ factors, and their interaction $(P=0.0105)$. The Tukey's multiple comparison test following two-way ANOVA determined significant tumor growth retardations in the groups of mice treated with a single dose (Ab Cocktail:1 Dose) or 4 doses (Ab Cocktail:4 Doses) of the 9-antibody cocktail in comparison to the mice of 3 control groups. Tumor growth for PBS (PBS Control:No Ab) and rabbit Ig control (Ig Control:1 Dose and Ig Control:4 Doses) groups of mice did not differ significantly.

Enhancement of survival by the antibody cocktail is shown as Kaplan Meier plots in Fig. 2B. Median survival was 16 days for PBS, 17 days for Ig control-1 dose, 22 days for 9-antibody cocktail-1 dose, 16 days for Ig control-4 doses and 26 days for 9-antibody cocktail-4 doses group. Long-rank (Mantel-Cox) test of the data revealed a highly significant $(P=0.0011)$ differences in the survival curves of the 4 groups of animals. Survival curves of antibody cocktail-treated groups were also significantly different from their respective Ig controls with a single dose $(P=0.0136)$ or 4 doses $(P=0.0141)$ of treatments. Survival curves for PBS control and Ig control groups did not differ significantly. The survival and hazard (MantelHaenszel) ratios between 1 dose Ig control and 9-antibody cocktail groups were 1.324 ( $95 \% \mathrm{Cl} 0.4269$ to 4.104) and 0.1264 ( $95 \% \mathrm{Cl} 0.02444$ to 0.6536 ), respectively. The survival and hazard ratios between 4 doses groups of Ig control and 9-antibody were 1.594 (95\% Cl 0.514-4.942) and 0.11 (95\% Cl 0.02148 to 4.942), respectively.

Animal Experiment 3: The results of combined treatment with PD1 inhibitor (PD1i) and antibody cocktail on tumor growth and mouse survival are presented in Fig. 3. The results show substantial retardation in tumor growth following combined treatment of antibody cocktail and PD1i in both 1 dose and 4 doses groups (Fig. 3A). Representative pictures of mice at 12 days post-tumor implantation are presented in Fig. 3B. Large tumors are seen in PBS control and PD1i alone groups. The representing mouse that received a single dose of antibody cocktail plus PD1i displays a small blush of pigmented tumor. The representative mouse that received 4 doses of antibody cocktail plus PD1i shows no visible tumor. Two-way ANOVA of the data in Fig. 3A showed significant tumor growth differences by the treatment $(P<0.001)$ and time $(P<0.001)$ factors, and their interaction $(P<0.001)$. The treatment with PD1i or rat isotype control of PD1i antibody alone did not produce any significant effects in comparison to PBS control. The data related to 
rat isotype control of PD1i antibody are presented to avoid over-crowding in the graph. Tukey's multiple comparison test following two-way ANOVA determined significant tumor growth retardations in the groups that received single and 4 doses of antibody cocktail plus PD1i compared to the PBS control $(P<0.0001), P D 1 i$ alone control $(P<0.0001)$, and respective Ig control groups with a single dose plus PD1i $(P<0.0001)$ or 4 doses plus PD1i $(P<0.0001)$ treatment.

Substantial enhancement of survival by the 1-dose and 4-dose antibody cocktail plus PD1 $\mathrm{i}$ is shown as Kaplan Meier plots in Fig. 4. Furthermore, $50 \%$ of the mice treated with 4 doses of antibody cocktail and PD1i had complete and durable responses. These mice developed normal fur and survived our entire observation period for more than six months. In contrast, all the animals in the control groups died in less than 25 days of tumor implantation. The median survival were 16.5 days for PBS control, 18.5 days for PBS+PD1i, 18.5 days for Ig control-1 dose+PD1i, 22.5 days for Ab cocktail-1 dose+PD1i, 28 days for Ig control-4 doses+PD1i and 56 days for Ab cocktail-4 doses+PD1i group. Log-rank (Mantel-Cox) test of the data revealed a highly significant $(P<0.0001)$ differences in the survival curves of the 6 groups of animals. The survival curves of the 1 and 4 dose antibody cocktail plus PD1i-treated groups were also significantly different from their respective Ig+PD1i control groups with a single dose $(P=0.0006)$ or 4 doses $(P=0.0006)$ of treatments. The survival curves of mice in PBS control, PD1i alone, isotype control of PD1i antibody (data not presented), and those in both Ig control+PD1i groups did not differ significantly. A high survival ratio of 1 dose 9-antibody cocktail+PD1i group 1.514 (95\% $\mathrm{Cl} 0.4881$ to 4.693) was observed in comparison to 1 dose Ig control+PD1i group. Mantel-Haenszel test showed a lower hazard ratio of 1 dose 9 -antibody cocktail+PD 1 i group $(0.04314,95 \% \mathrm{Cl} 0.00724$ to 0.2571$)$ compared to 1 dose Ig control+PD1i group. The survival and hazard ratios between 4 doses groups of Ig control and 9-antibody were 2.605 ( $95 \% \mathrm{Cl} 0.651$ to 10.41 ) and 0.0464 (95\% Cl 0.00803 to 0.268 ), respectively.

Animal Experiment 4: Fig. 5 shows that 6.5 months after a complete response to 4 doses of antibody cocktail and PD1i (Fig. 4), these mice were persistently resistant to re-inoculation without any further treatment. It is highly unlikely than any of the rabbit antibodies were present at the time of re-inoculation. It appears that following the initial treatment and complete response, these mice developed a robust antimelanoma adaptive immune response. Fig. 5 includes a set of normal untreated mice for comparison.

\section{Discussion}

In this study, we targeted multiple tumor-specific proteins that were not overexpressed driver mutations. These are the mutations largely ignored relative to cancer biology and cancer treatment. They are the mutational dark matter that are highly abundant in most if not all cancers. Rabbits vaccinated with peptides representing the mutated region of these tumor-specific proteins resulted in high-affinity polyclonal antibodies. Individually, these antibodies had heterogeneous binding to B16-F10 cells. This reflects the variable and unpredictable expression of these proteins. However, combining the antibodies 
together as a cocktail resulted in strong homogeneous binding. These results support the idea that multiple antibodies to multiple tumor-specific neoantigens can potentially overcome fundamental limitations associated with tumor heterogeneity. This idea is further supported by tumor inhibition experiments. A single dose of the cocktail of antibodies significantly inhibited tumor growth and prolonged survival of mice implanted with B16-F10 melanoma cells. Four doses substantially increased both tumor inhibition and survival.

PD1 inhibition of mouse melanoma has been reported in multiple studies. Across a range of treatment schedules and differing quantities of tumor cells inoculated, the ability of anti-PD1 antibody to inhibit growth of B16-F10 melanoma cells has been modest [18-20]. We observed a similar result and PD1 inhibition had minimal impact on tumor growth and survival. However, we observed that combining the cocktail of antibodies with anti-PD1 antibody resulted in substantial inhibition of tumor growth and prolongation of survival. Indeed, combination of four doses of the cocktail of antibodies with PD1 inhibition permanently prevented any melanoma growth in $50 \%$ of the mice. Overall these remarkable results show both independent actions of the antibodies and integration with an additional form of immunotherapy.

Selection of mutated targets was based upon published NGS sequence data of B16-F10 melanoma cells [13]. We did not attempt to select for driver mutations. We did not select based upon expression data. The only criteria were that the mutation was related to the cell surface by its reported presence in the membrane or as a secreted protein. For membrane proteins we restricted the selection to those with mutations not in the cytoplasmic or intramembrane portion. These selection criteria retrieved many possible targets. We designed short peptides representing the mutated section of the protein with the mutated amino acid in the center. The peptide design was based on our previous successful vaccination experiments with mice [15]. The set of peptides predicted to be immunogenic in the rabbit were then synthesized and used as vaccines. All of the 9 selected peptides produced high titers of antibodies. Affinity enrichment yielded 4 to $6 \mathrm{mg}$ of high-affinity polyclonal antibodies. These simple selection criteria were surprisingly productive at producing desirable antibodies. The majority bound B16-F10 cells and when combined together produced remarkable tumor inhibition of a relatively aggressive tumor.

To assess the possible applicability of our approach for treating cancer patients we analyzed the single non-synonymous mutations of four breast cancer patients from published sequence data to determine how many mutations were membrane-associated [21]. The total number of gene mutations in these patients ranged from 15 to 221 . We determined the cell location of each identified gene mutation using the human protein atlas project [22] or the human gene database through GeneCards [23]. We found that $27 \%-46 \%$ of all the nonsynonymous mutations reported in these breast cancer patients were proteins found on the plasma membrane. This information indicates that cancer cell surface mutations in breast cancer patients are not uncommon and will be potentially available for targeting with antibodies.

We observed no adverse events in the treated mice. Antibodies, even from different species, are not inherently toxic [24-26]. The strategy of using tumor-specific mutations may reduce cross-binding to 
normal proteins. Using a cocktail which diminishes the dose of each antibody may further diminish possible normal cell toxicity to any individual antibody. A normal cell that expresses a cross-reactive antigen would be exposed to a relatively low dose of that individual antibody. The likelihood of normal cells binding to additional antibodies from the cocktail becomes increasingly remote as the number of different antibodies in the cocktail increases.

Cell surface mutations that are not overexpressed or involved in maintaining the malignant phenotype have been largely ignored as potential targets for therapy. It appears that such mutations are in abundance. It also appears that there is considerable variation between patients in which proteins are mutated. These types of mutations would normally be considered of low value from the perspective of developing single pharmaceutical reagents that can be used for many patients.

However, the success rate for curing stage IV cancer patients using the conventional strategy of a single antibody targeting a single overexpressed protein is close to zero. Despite published lists of desirable targets to help guide researchers, the search for a single overexpressed universal target in cancer patients is elusive likely does not exist $[27,28]$. We propose a new approach that if successful will fundamentally change how patients will be treated with antibodies. This will be accomplished by using multiple antibodies that bind multiple tumor-specific antigens resulting in dense homogeneous binding of all of the cancer cells. Antibodies are highly bioactive and readily induce cancer cell cytotoxicity as long as antibody binding achieves a critical threshold. In clinical practice, however, monoclonal antibodies never functionally achieve critical threshold binding on the full population of heterogeneous cancer cells. Targeting the unique set of tumor-specific mutations present in a patient's cancer by using multiple antibodies could possibly overcome this limitation of single-target monoclonal antibodies.

\section{Conclusions}

Our data shows that pooling multiple anti-neoantigen antibodies results in intense and homogenous antibody binding across the cell population. Remarkable inhibition of tumor growth was observed with pooled antibody treatment. Even a single treatment inhibited growth of an aggressive tumor. Four treatments prevented tumor growth in $50 \%$ of the mice. The response was durable and rendered the responding mice resistant to subsequent tumor challenge.

\section{Declarations}

Ethics approval and consent to participate: All animal procedures in the present study were approved (Protocol \# 18-002) by the University of Vermont Institutional Animal Care and Use Committee (UVM IACUC).

Consent for publication: Not applicable. 
Availability of data and material: Not applicable.

Competing interests: The authors declare that they have no competing interests.

Funding: This work was supported by the SD Ireland Cancer Research Fund, Cast Off Chemo Foundation, and the Department of Surgery at the University of Vermont Larner College of Medicine internal grant. The funding bodies had no role in the design of the study and collection, analysis and interpretation of the data, and in writing of the manuscript.

Authors' contributions: GS designed the studies, helped acquire the data, interpreted the data, assisted in writing the manuscript, and is personally accountable for this work. YS helped acquire the data, interpreted the data, assisted in writing the manuscript, and is personally accountable for this work. SP helped acquire the data, interpreted the data, assisted in writing the manuscript, and is personally accountable for this work. DK designed the studies, interpreted the data, assisted in writing the manuscript, and is personally accountable for this work.

Acknowledgements: Thanks to Linda Mei for her assistance in the submission of this manuscript.

\section{References}

1. Slamon DJ, Leyland-Jones B, Shak S, Fuchs H, Paton V, Bajamonde A, Fleming T, Eiermann W, Wolter J, Pegram M, Baselga J, Norton L (2001) Use of chemotherapy plus a monoclonal antibody against HER2 for metastatic breast cancer that overexpresses HER2. N Engl J Med 344 (11):783-792. doi:10.1056/NEJM200103153441101

2. Alexander P, Connell DI, Mikulska ZB (1966) Treatment of a murine leukemia with spleen cells or sera from allogeneic mice immunized against the tumor. Cancer Res 26 (7):1508-1515

3. Blair PB (1968) Immunology of the mouse mammary tumor virus (MTV): neutralization of MTV by mouse antiserum. Cancer Res 28 (1):148-149

4. Cihak J, Ziegler HW, Kolsch E (1981) Regulation of immune responses against the syngeneic ADJPC-5 plasmacytoma in BALB/c mice. I. Analysis of immune parameters involved. Immunology 43 (1):133-143

5. Lindenmann J, Klein PA (1965) Immunity to Transplantable Tumors Following Viral Oncolysis.li. Antigenic Similarities between Three Unspecific Mouse Tumors. J Immunol 94:461-466

6. Mu B, Yang JL, Gou LT, Yao YQ, Zhou Y, Cheng ZH, Shi HS, Li ZY, Wen Y, Leng F, Cui FY, Ma TT, Wei YQ (2011) Polyclonal rabbit anti-murine plasmacytoma cell globulins induce myeloma cells apoptosis and inhibit tumour growth in mice. Apoptosis 16 (4):370-381. doi:10.1007/s10495-010-0568-7

7. Sell S, Sheppard HW, Jr., Nickel R, Stillman D, Michaelsen M (1976) Effect of anti-alpha1-fetoprotein on alpha1-fetoprotein-producing rat tumors in vivo and in vitro. Cancer Res 36 (2 Pt 1):476-480

8. Hubert P, Heitzmann A, Viel S, Nicolas A, Sastre-Garau X, Oppezzo P, Pritsch O, Osinaga E, Amigorena S (2011) Antibody-dependent cell cytotoxicity synapses form in mice during tumor-specific antibody immunotherapy. Cancer Res 71 (15):5134-5143. doi:10.1158/0008-5472.CAN-10-4222 
9. Penfold PL, Greenberg AH, Roitt IM (1976) Characteristics of the effector cells mediating cytotoxicity against antibody-coated target cells. III. Ultrastructural studies. Clin Exp Immunol 23 (1):91-97

10. Moller E, Moller G (1962) Quantitative studies of the sensitivity of normal and neoplastic mouse cells to the cytotoxic action of isoantibodies. J Exp Med 115:527-553

11. Zhang S, Helling F, Lloyd KO, Livingston PO (1995) Increased tumor cell reactivity and complementdependent cytotoxicity with mixtures of monoclonal antibodies against different gangliosides. Cancer Immunol Immunother 40 (2):88-94

12. Glassy MC, McKnight ME, Kotlan B, Glassy EF, Koda K (2007) Cocktails of human anti-cancer antibodies show a synergistic effect in nude mouse tumor xenografts. Hum Antibodies 16 (3-4):8798

13. Castle JC, Kreiter S, Diekmann J, Lower M, van de Roemer N, de Graaf J, Selmi A, Diken M, Boegel S, Paret C, Koslowski M, Kuhn AN, Britten CM, Huber C, Tureci O, Sahin U (2012) Exploiting the mutanome for tumor vaccination. Cancer Res 72 (5):1081-1091. doi:10.1158/0008-5472.CAN-113722

14. El-Manzalawy Y, Dobbs D, Honavar V (2008) Predicting linear B-cell epitopes using string kernels. J Mol Recognit 21 (4):243-255. doi:10.1002/jmr.893

15. Shukla GS, Sun YJ, Pero SC, Sholler GS, Krag DN (2018) Immunization with tumor neoantigens displayed on $\mathrm{T} 7$ phage nanoparticles elicits plasma antibody and vaccine-draining lymph node B cell responses. J Immunol Methods 460:51-62. doi:10.1016/j.jim.2018.06.009

16. Shukla GS, Krag DN, Peletskaya EN, Pero SC, Sun YJ, Carman CL, McCahill LE, Roland TA (2013) Intravenous infusion of phage-displayed antibody library in human cancer patients: enrichment and cancer-specificity of tumor-homing phage-antibodies. Cancer Immunol Immunother 62 (8):13971410. doi:10.1007/s00262-013-1443-5

17. Shukla GS, Pero SC, Sun YJ, Carman CL, Harlow S, Krag DN (2018) Characterization of sentinel nodederived antibodies from breast cancer patients. J Immunol Methods 455:14-23. doi:10.1016/j.jim.2018.01.010

18. Curran MA, Montalvo W, Yagita H, Allison JP (2010) PD-1 and CTLA-4 combination blockade expands infiltrating $T$ cells and reduces regulatory $T$ and myeloid cells within B16 melanoma tumors. Proc Natl Acad Sci U S A 107 (9):4275-4280. doi:10.1073/pnas.0915174107

19. McGray AJ, Hallett R, Bernard D, Swift SL, Zhu Z, Teoderascu F, Vanseggelen H, Hassell JA, Hurwitz AA, Wan Y, Bramson JL (2014) Immunotherapy-induced CD8+ T cells instigate immune suppression in the tumor. Mol Ther 22 (1):206-218. doi:10.1038/mt.2013.255

20. Twyman-Saint Victor C, Rech AJ, Maity A, Rengan R, Pauken KE, Stelekati E, Benci JL, Xu B, Dada H, Odorizzi PM, Herati RS, Mansfield KD, Patsch D, Amaravadi RK, Schuchter LM, Ishwaran H, Mick R, Pryma DA, Xu X, Feldman MD, Gangadhar TC, Hahn SM, Wherry EJ, Vonderheide RH, Minn AJ (2015) Radiation and dual checkpoint blockade activate non-redundant immune mechanisms in cancer. Nature 520 (7547):373-377. doi:10.1038/nature14292 
21. Kato T, Park JH, Kiyotani K, Ikeda Y, Miyoshi Y, Nakamura Y (2017) Integrated analysis of somatic mutations and immune microenvironment of multiple regions in breast cancers. Oncotarget 8 (37):62029-62038. doi:10.18632/oncotarget.18790

22. Uhlen M, Fagerberg L, Hallstrom BM, Lindskog C, Oksvold P, Mardinoglu A, Sivertsson A, Kampf C, Sjostedt E, Asplund A, Olsson I, Edlund K, Lundberg E, Navani S, Szigyarto CA, Odeberg J, Djureinovic D, Takanen JO, Hober S, Alm T, Edqvist PH, Berling H, Tegel H, Mulder J, Rockberg J, Nilsson P, Schwenk JM, Hamsten M, von Feilitzen K, Forsberg M, Persson L, Johansson F, Zwahlen M, von Heijne G, Nielsen J, Ponten F (2015) Proteomics. Tissue-based map of the human proteome. Science 347 (6220):1260419. doi:10.1126/science.1260419

23. Stelzer G, Rosen N, Plaschkes I, Zimmerman S, Twik M, Fishilevich S, Stein TI, Nudel R, Lieder I, Mazor Y, Kaplan S, Dahary D, Warshawsky D, Guan-Golan Y, Kohn A, Rappaport N, Safran M, Lancet D (2016) The GeneCards Suite: From Gene Data Mining to Disease Genome Sequence Analyses. Curr Protoc Bioinformatics 54:1 30 31-31 30 33. doi:10.1002/cpbi.5

24. Burkhalter F, Schaub S, Bucher C, Gurke L, Bachmann A, Hopfer H, Dickenmann M, Steiger J, Binet I (2016) A Comparison of Two Types of Rabbit Antithymocyte Globulin Induction Therapy in Immunological High-Risk Kidney Recipients: A Prospective Randomized Control Study. PLoS One 11 (11):e0165233. doi:10.1371/journal.pone.0165233

25. Ali AK (2013) Pharmacovigilance Analysis of Serious Adverse Events Reported for Biologic Response Modifiers Used as Prophylaxis against Transplant Rejection: a Real-World Postmarketing Experience from the US FDA Adverse Event Reporting System (FAERS). Int J Organ Transplant Med 4 (2):62-71

26. Klein JL, Sandoz JW, Kopher KA, Leichner PK, Order SE (1986) Detection of specific anti-antibodies in patients treated with radiolabeled antibody. Int J Radiat Oncol Biol Phys 12 (6):939-943

27. Cheever MA, Allison JP, Ferris AS, Finn OJ, Hastings BM, Hecht TT, Mellman I, Prindiville SA, Viner JL, Weiner LM, Matrisian LM (2009) The prioritization of cancer antigens: a national cancer institute pilot project for the acceleration of translational research. Clin Cancer Res 15 (17):5323-5337. doi:10.1158/1078-0432.CCR-09-0737

28. Orentas RJ, Yang JJ, Wen X, Wei JS, Mackall CL, Khan J (2012) Identification of cell surface proteins as potential immunotherapy targets in 12 pediatric cancers. Front Oncol 2:194. doi:10.3389/fonc. 2012.00194

\section{Tables}


Table 1 List of the peptides that were derived from the selected mutated B16-F10 tumor cell proteins and used for rabbit polyclonal antibodies production.

\begin{tabular}{lcll}
\hline \multicolumn{1}{c}{ Peptide ID } & Mutation & Mutated epitope & \multicolumn{1}{c}{ Normal epitope } \\
\hline Cd9912 & P86Q & GTTRAQSNPME & GTTRAPSNPME \\
Fgfbp1 & R26G & EKVRKGAKNAP & EKVRKRAKNAP \\
\hline Lama1 & D656N & NFRDFNTRREI & NFRDFDTRREI \\
\hline Ush2a & L4514P & SVLSPPVKGQT & SVLSPLVKGQT \\
\hline Psg17 & T340I & RSRREIVYTNG & RSRRETVYTNG \\
\hline Psg25 & R102S & RETLHSNGSLW & RETLHRNGSLW \\
Serpinc1 & L395F & GGRDDFYVSDA & GGRDDLYVSDA \\
\hline Tpo(II) & D656G & KQMKALRGGDR & KQMKALRDGDR \\
\hline Ptgfrn & T620P & KLENWPDASRV & KLENWTDASRV \\
\hline
\end{tabular}

Peptide IDs represented by the gene names respective to mutated proteins. Mutation column shows amino acid substitution with its position in the protein sequence. The mutated and corresponding normal amino acids in the selected peptides are presented in bold red and blue fonts, respectively.

\section{Figures}


A
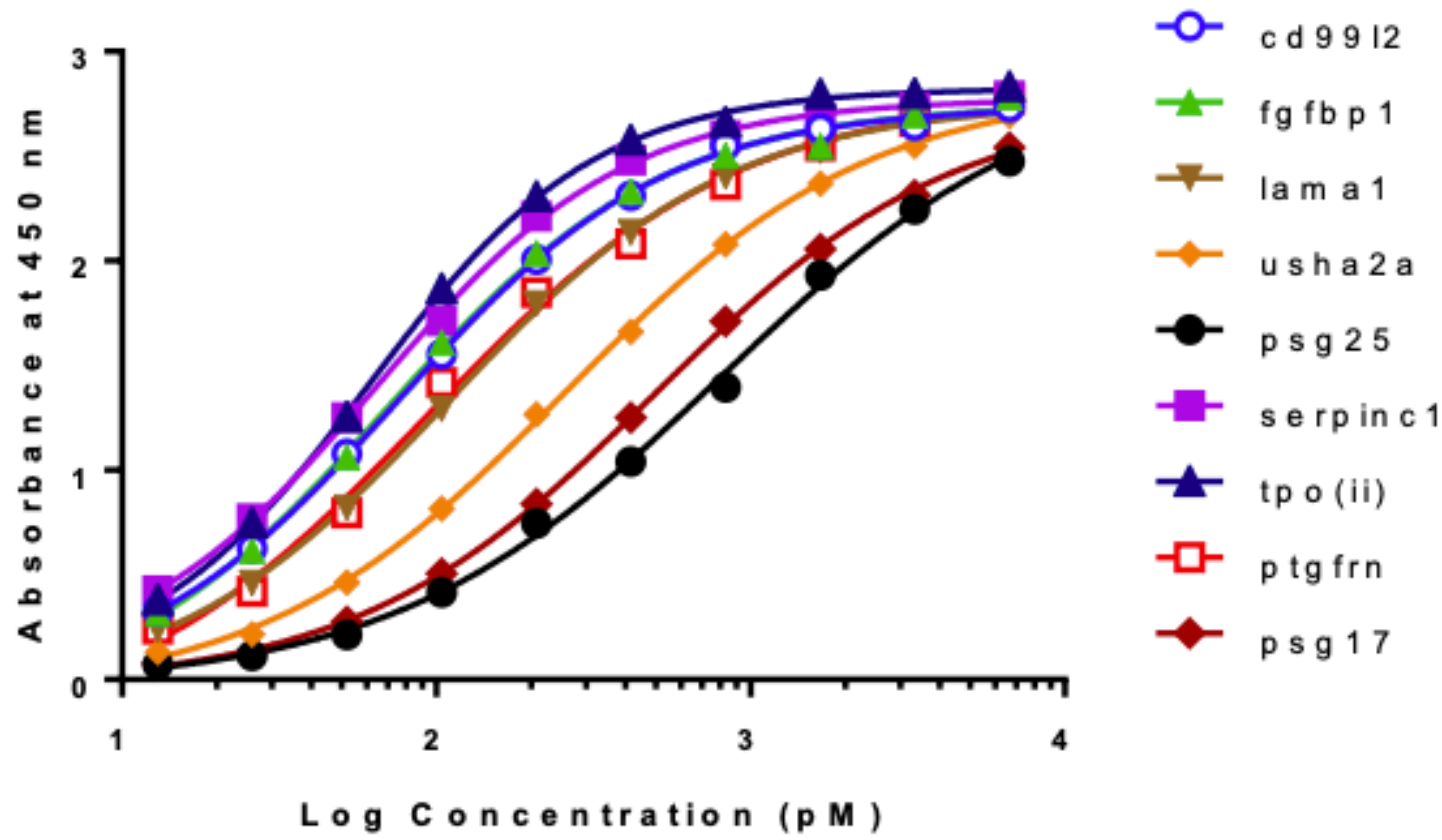

B

\begin{tabular}{|c|c|c|c|c|c|c|c|c|c|}
\hline Abs & cd9912 & fgfbp1 & lama1 & usha2a & psg25 & serpinc1 & tpo(ii) & ptgfrn & psg17 \\
\hline$M$ & $7.6 \mathrm{E}-11$ & $6.9 \mathrm{E}-11$ & $1.1 \mathrm{E}-10$ & $2.5 \mathrm{E}-10$ & $8.0 \mathrm{E}-10$ & $6.4 \mathrm{E}-11$ & $6.1 \mathrm{E}-11$ & $9.4 \mathrm{E}-10$ & $4.9 \mathrm{E}-10$ \\
\hline
\end{tabular}

C

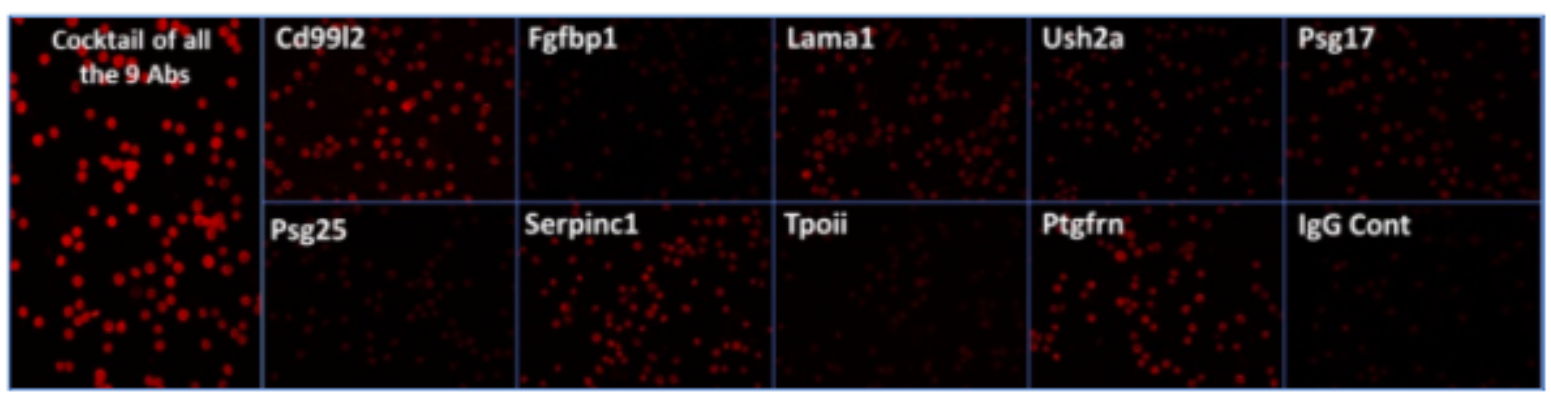

Figure 1

Characterization of rabbit polyclonal antibodies raised against selected mutated peptides. (A) Binding curves of serially diluted (2-fold) samples of individual rabbit anti-peptide antibodies. Each absorbance value plotted for different antibody samples represents average values from duplicate $(\leq 5 \%)$ wells. (B) EC50 of affinity purified rabbit polyclonal antibodies raised against selected mutated peptides. (C) Binding of the 9 anti-peptide antibodies to B16F10 melanoma cells by fluorescence microscopy. Far left panel is cocktail of all 9 rabbit antibodies. Each of the other panels represent binding with each antipeptide antibody to the indicated neoantigen. The lower right corner micrograph, which represents rabbit IgG control (without an anti-peptide antibody), shows background binding to B16-F10 cells. 
A

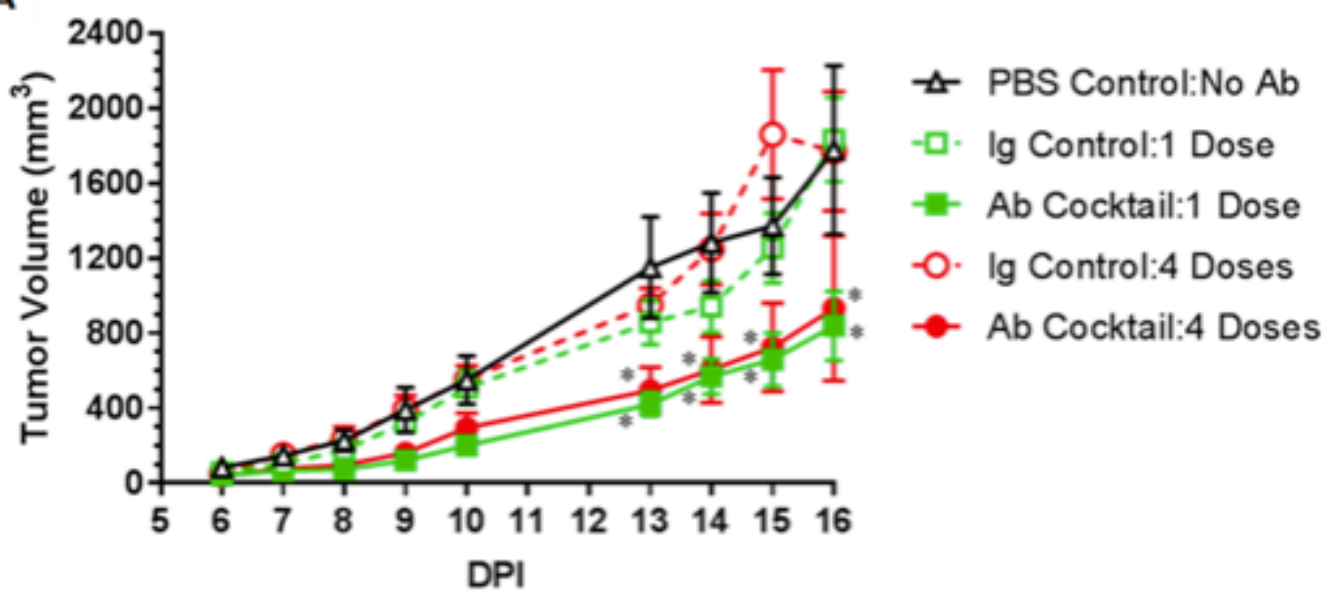

\begin{tabular}{lccccc}
\hline ANOVA Table & SS & DF & MS & $F(D F n, D F d)$ & $P$ value \\
\hline Interaction & 7438064 & 32 & 232440 & $F(32,219)=1.754$ & $P=0.0105$ \\
Time & 56164807 & 8 & 7020601 & $F(8,219)=52.98$ & $P<0.0001$ \\
Treatment & 10314255 & 4 & 2578564 & $\mathrm{~F}(4,219)=19.46$ & $\mathrm{P}<0.0001$ \\
\hline
\end{tabular}

B

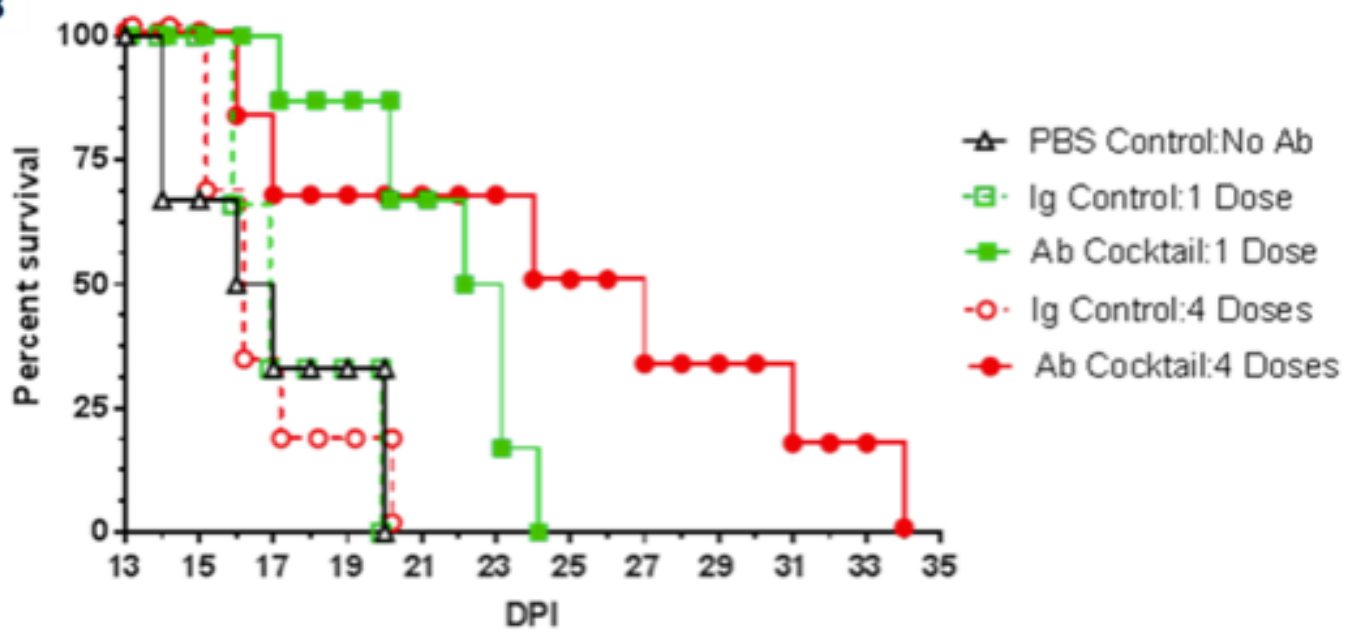

\begin{tabular}{lcccccc} 
Log-rank (Mantel-Cox) test \\
\hline Comparisons & Chi squire & df & P value & Median survival Survival ratio & $95 \% \mathrm{Cl}$ \\
\hline All curves & 18.21 & 4 & 0.0011 & & & \\
Ab Cocktail 1 dose vs Ig Control:1 dose & 6.087 & 1 & 0.0136 & 17.0 vs 22.5 d & 1.324 & 0.427 to 4.104 \\
Ab Cocktail:4 doses vs lg Control:4 doses & 7.023 & 1 & 0.0080 & 16.0 vs 25.5d & 1.594 & 0.514 to 4.942 \\
\hline
\end{tabular}

\section{Figure 2}

The effects of treatment of B16-F10 melanoma tumor bearing mice with a cocktail of 9 antibodies against B16-F10 tumor-derived mutated peptides. (A) The antibody cocktail treatment retarded the tumor growth in comparison to that in the control mice groups. Two-way ANOVA analysis of the data show significantly different curves by treatment, time and interaction. *Significantly different $(P \leq 0.05)$ in comparison to PBS and respective Ig control groups as determined by Tukey's multiple comparisons test. The values represent mean \pm SEM of six or surviving animals in each group. (B) The treatment of tumor bearing mice with the cocktail of 9 antibodies significantly increased the survival of mice. The median survival were 16.5 days for PBS control, 17 days for Ig control-1 dose, 22.5 days for 9-antibody cocktail-1 dose, 16 days for days for Ig control-4 doses and 25.5 days for 9-antibody cocktail-4 doses group. DPI=Days post-implantation. 
A

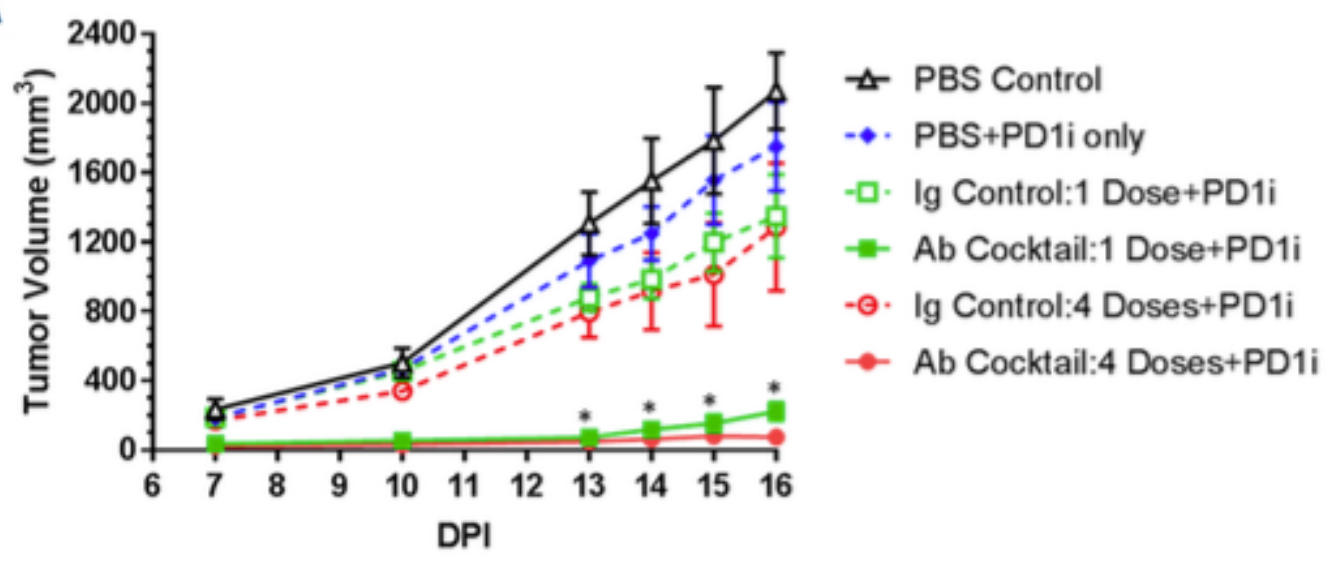

\begin{tabular}{lccccc}
\hline ANOVA Table & SS & DF & MS & $F(D F n, D F d)$ & P value \\
\hline Interaction & 12295685 & 25 & 491827 & $\mathrm{~F}(25,180)=3.495$ & $\mathrm{P}<0.0001$ \\
Time & 26177154 & 5 & 5235431 & $\mathrm{~F}(5,180)=37.2$ & $\mathrm{P}<0.0001$ \\
Treatment & 43325070 & 5 & 8665014 & $\mathrm{~F}(5,180)=61.57$ & $\mathrm{P}<0.0001$ \\
\hline
\end{tabular}

B

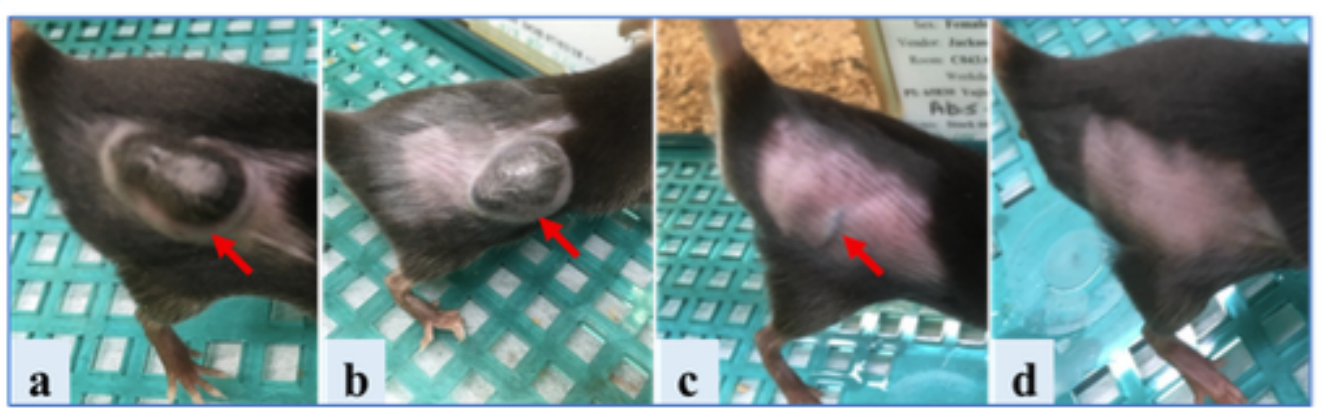

Figure 3

The effects of combined treatment of B16-F10 tumor bearing mice with PD1 inhibitor (PD1i) and the cocktail of 9 antibodies against B16-F10 tumor-derived mutated peptides on tumor growth. (A) The treatment with a 9-antibody cocktail+PD1i retarded the tumor growth in comparison to that in the control groups of mice. Two-way ANOVA analysis of the data show significantly different curves by treatment, time and interaction. *Tukey's multiple comparisons test determined that both the antibody cocktail+PD1 i-treated groups were significantly different $(\mathrm{P} \leq 0.05)$ in comparison to PBS and/or respective Ig control groups. The values represent mean \pm SEM of six or surviving animals in each group. DPI=Days post-implantation. (B) Representative pictures of mice at 12 days post-tumor implantation. Big tumors are seen in (a) PBS control and (b) PD1 inhibitor alone groups; (c) mouse that received a single dose of antibody cocktail+PD1i displays a small blush of pigmented tumor; (d) representative mouse that shows no visible tumor following 4 doses of antibody cocktail+PD1i. DPI=Days post-implantation. 


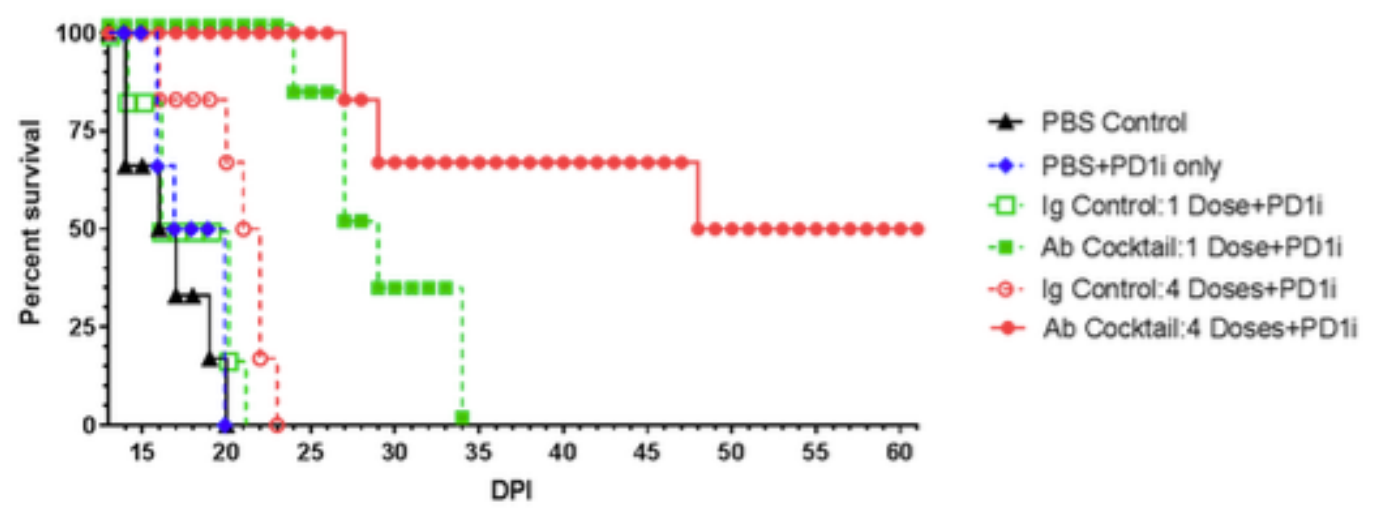

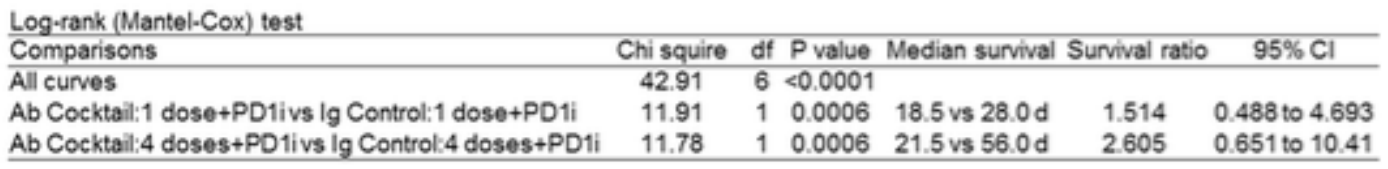

\section{Figure 4}

The combined treatment of B16-F10 melanoma tumor bearing mice with the cocktail of 9 anti-peptide antibodies and PD1 inhibitor (PD1i) significantly increased the survival of mice. Survival curves of the 9antibody cocktail+PD1i-treated groups were significantly different from the control groups. The median survivals were 16.5 days for PBS control, 18.5 days for PBS+PD1i, 18.5 days for Ig control-1 dose+PD1i, 22.5 days for Ab cocktail-1 dose+PD1i, 28 days for lg control-4 doses+PD1i and 56 days for Ab cocktail-4 doses+PD1i group. DPI=Days post-implantation.

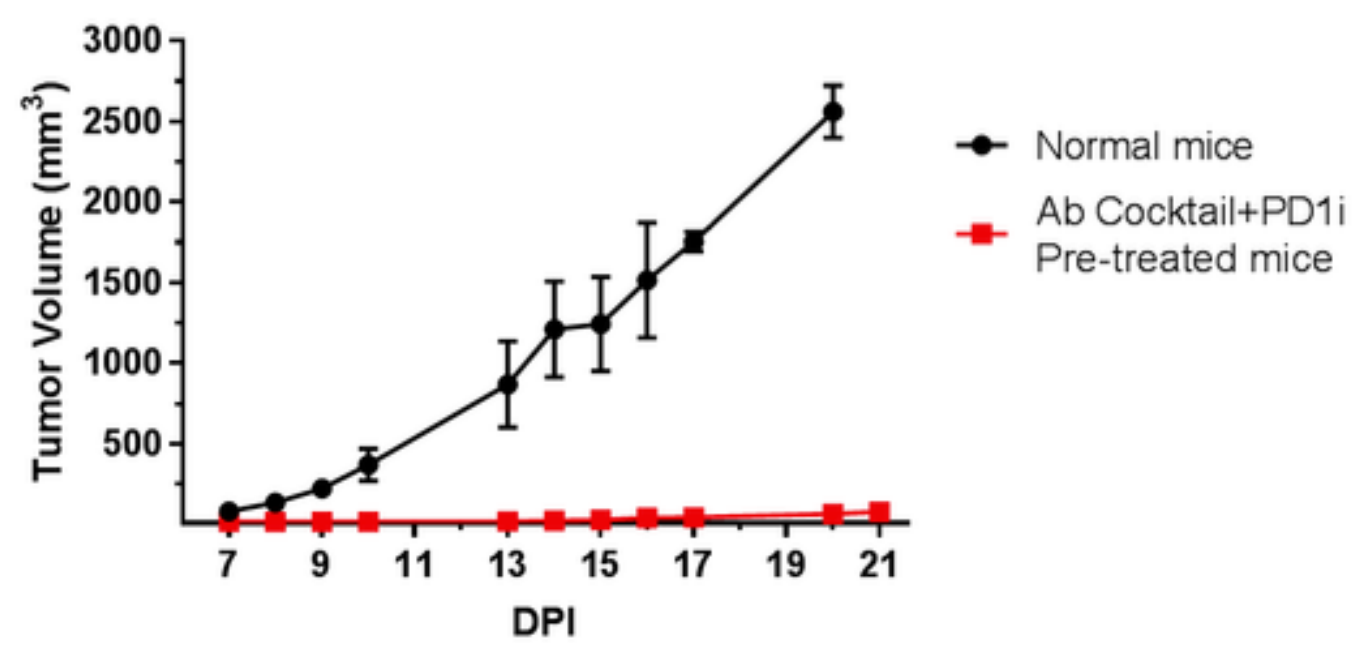

\section{Figure 5}

Tumor growth in the mice that had a durable complete response to tumor challenge (Fig. 4) for 6.5 months were re-challenged with 3x105 B16-F10 cells. A set of normal mice were inoculated with 3x105 B16-F10 cells in the same way for a comparison. Mice with a previous durable response had resistance to the tumor inoculation. Untreated control mice had rapid growth of tumor with no survival at $21 \mathrm{DPI}$. Each 
data represents mean \pm SEM of tumor volumes of normal ( $n=6$ up to $14 \mathrm{DPI}, n=5$ up to $16 \mathrm{DPI}, \mathrm{n}=3$ up to $20 \mathrm{DPI})$ and pre-treated $(\mathrm{n}=3)$ mice.

\section{Supplementary Files}

This is a list of supplementary files associated with this preprint. Click to download.

- ARRIVEGuidelinesChecklist.pdf 